\title{
An Empirical Mode Decomposition Approach to Peak Load Demand Forecasting
}

\author{
Victor Okolobah and Zuhaimy Ismail \\ Department of Mathematical Sciences, Universiti of Teknologi Malaysia, Johor Bahru, Johor, Malaysia; \\ dynamic4vic@yahoo.com, zuhaimy@utm.my
}

\begin{abstract}
An accurate and reliable electric load forecasting model is very essential for efficient and effective operation of the Electricity Supply Industry (ESI). Several single models have been developed for electric load forecast for ESI but it is becoming increasingly difficult to obtain accurate forecast by these models because of the volatility coupled with the nonlinear and non- stationary nature of electric load series. In this paper, we propose a novel Electric Peak load forecasting model that combines empirical mode decomposition (EMD) and artificial neural network (ANN). The propose model involves three stages of development. In the first stage, the historical load data obtained from Power holding company of Nigeria (PHCN), Bida is decomposed into several intrinsic mode functions and a residue component using the EMD sifting process. The second stage involves building separate neural network models for each of these IMFS and residue component and the last stage involves combining the predictions from these models and making forecast. When the forecast from this model is compared with that obtained from a conventional neural network model, it was observed that the proposed model outperforms the conventional neural network model, by $2.3 \%$ for the whole year model and by $1.8 \%$ for the weekday model, judging by the forecast accuracy of both models.
\end{abstract}

Keywords: Empirical Mode Decomposition, Intrinsic Mode Function, Sifting, Model, Forecasting.

\section{Introduction}

Over the last two decades, many developing nations have taken steps towards revamping its energy sector particularly the electric power sector. For this reason, many countries, electricity supply industry have evolved many models for forecasting its load demand.

Given the importance of forecasting load demand, there have been many studies in the literatures that discuss electricity load demand in Nigeria, this include $[1,2,3]$. Furthermore, Adepoju et al. [4], and Adenikinju [5] modeled the Short Term Load Forecasting (STLF) for Nigeria using the Artificial Neural Network (ANN). However, there are several uncertainties involved in load forecasting and also many more issues in STLF which has not been considered. Nonetheless, to our knowledge there have been little published records in the literatures that attempt to forecast load demand in Nigeria that hybrids any two methods.

\footnotetext{
* Corresponding author:
}

Zuhaimy Ismail (zuhaimy@utm.my)
In the light of this, we attempt to present a technique for STLF that combines Empirical Mode Decomposition (EMD) and Artificial Neural Network (ANN). The motivation for this is to explore the time series decomposition attribute of EMD [6]. We first decompose the historical load data into several Intrinsic Mode Functions (IMFs) of different frequency levels. These IMFs are then used to develop several neural network models that predict these IMFs. Collecting these predictions together produces the forecast of the proposed model.

The remaining part of this paper is structured as follows: Section 2 is the methodology in which we discuss the EMD technique extensively followed by the discussion of ANN models. The section closes by discussing the proposed model EMD-ANN. Section 3 concerns itself with implementation of the proposed model on a real life data taken from a branch of Power Holding Company of Nigeria (PHCN). Section 4 is discussion of results while the 
conclusion is presented in section 5. Section 6 is acknowledgement in which we appreciated the contributions of a few individuals and organizations towards making this research work come to limelight.

\section{Methodology}

In this section, we attempt to discuss the key components of the proposed forecasting method, that is, EMD-ANN. We first discuss the EMD extraction process extensively, then we discuss the ANN model that will be combined with the EMD and lastly, we present a comprehensive description of the proposed model.

\subsection{Empirical Mode Decomposition (EMD)}

EMD is an adaptive time series decomposition technique (Huang et al., 1998). The main idea behind the EMD extraction process called sifting [7] is the decomposition of data into several signals that are themselves stationary. These signals are called Intrinsic Mode Functions (IMFs). These signals vary from those with high frequencies to those with low frequencies, for instance IMF1 is of higher frequency than IMF2 and IMF2 is of a higher frequency than IMF3 and so on up to IMFn which usually is the residue. EMD method has extensively been applied in the field of load forecasting in many studies in recent times as in $[8,9,10]$. For a function to qualify as an IMF it must satisfy the following two conditions [11]:

I. The mean value of the upper envelope defined by the local maxima and the lower envelope defined by the local minima must be zero.

II. The number of the local minima must differ by at most one.

\subsubsection{The EMD Algorithm}

The algorithm for the EMD extraction process can be summarized into five steps $[12,13]$.

Step 1: Identify all extrema in the load data $X_{t}$ and connect them by a cubic spline line. Denote the upper envelope as $e_{\max (t)}$ and the lower envelope as $e_{\min (t)^{\circ}}$

Step 2: Obtain the mean of the upper and lower envelopes that is

$$
M(t)=e_{\max (t)}+\frac{e_{\min (t)}}{2}
$$

Step 3: subtract the mean from the original series and denote as $d(t)$

$$
d(t)=X(t)-M(t)
$$

Step 4: Evaluate $d(t)$

- If $\mathrm{d}(\mathrm{t})$ satisfies the conditions of being an IMF as outlined above, then denote it as the ith IMF and replace $\mathrm{X}(\mathrm{t})$ with the residue $r(t)$ which is:

$$
r(t)=X(t)-d(t)
$$

The $\mathrm{i}^{\text {th }}$ IMF is often denoted as $C_{i}(t)$ and $I$ is called its index [19].

- If $d(t)$ is not an IMF in other words does not satisfy the conditions of being IMF the replace $X(t)$ with $d(t)$.

Step 5: Repeat steps 1 to 4 until the desired stopping criterion is reached by the residue.

As emphasized by Chen et al. [14] the stopping criterion is a very important step in the EMD process. Several stopping criteria have been proposed in the literatures. For the different stopping criteria that can be employed see $[15,16$, 17]. For this study, we used the stopping criteria proposed by Huang et al. [16] which is based on zero crossings and when the extrema does not differ by more than one. The EMD sifting process described here is shown in Figure 1.

\subsection{Artificial Neural Network (ANN).}

ANN are non-linear mapping structures which are based on the function of the human brain. ANN are very good tools for modeling when the underlying relationship inherent in the data is unknown. ANN learns, identifies and correlates patterns between input and corresponding datasets. After training, ANN can be used to predict the outcome of an independent input data [18]. As a result of this, ANN can be employed in modeling load data which are often non-linear and non-stationary. Figure 2 is a schematic representation of a feed-forward network.

Processing elements in an ANN are called neurons. Neurons are interconnected by information channels called interconnections. Each neuron can have multiple inputs but can only have one output. Inputs to a neuron can be from external stimuli or could be the output of other neurons. The backpropagation learning algorithm is the most common of all methods used in training a neural network and is the method employed in developing the proposed hybrid model discussed in this paper. The backpropagation algorithm is discussed below. 


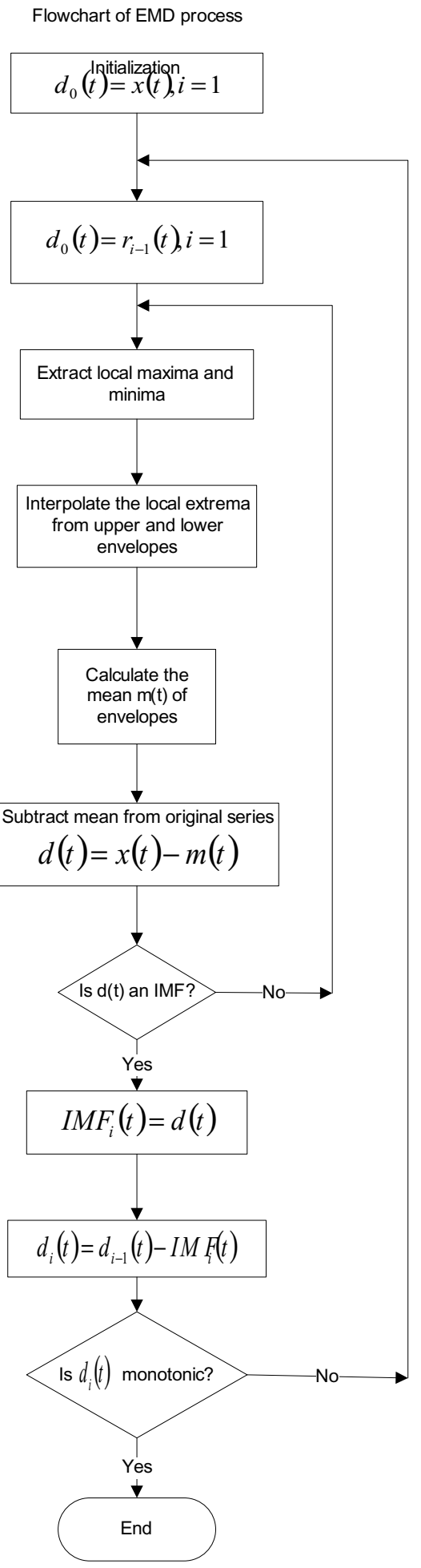

Figure 1. EMD Extraction Process.

\subsubsection{Backpropagation Algorithm [18]}

The multilayer network containing hidden units is trained using a supervised learning algorithm (a network in which

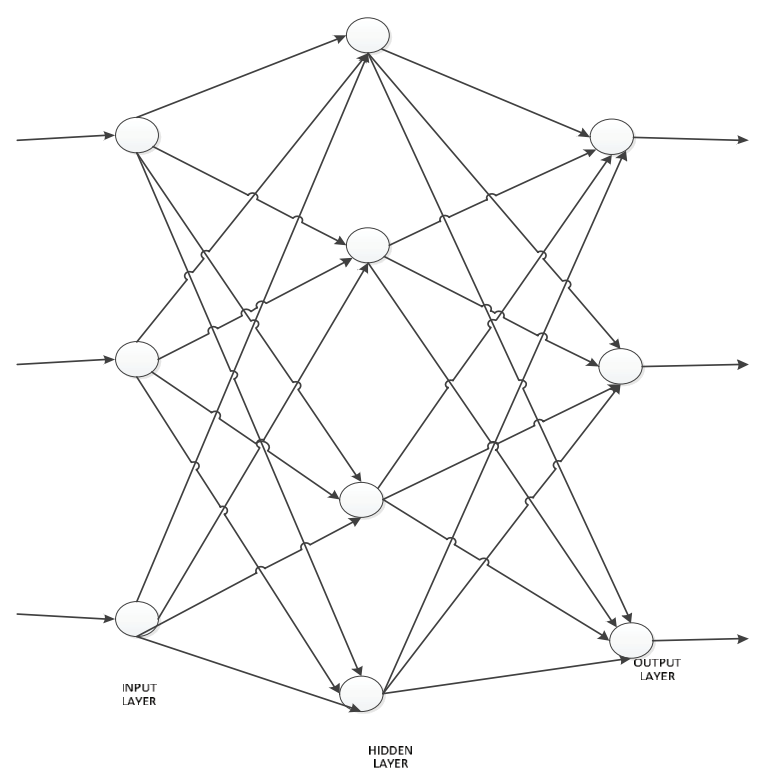

Figure 2. Schematic 3 layers feed-forward network.

each output unit is informed before-hand what its desired input ought to be) is a good example of the backpropagation. The network uses the data to adjust its weights and thresholds so that a minimum error of its prediction of the training dataset can be obtained. Let $W_{\mathrm{ji}}$ be the weight of the connection from unit $U_{\mathrm{j}}$ to unit $U_{\mathrm{i}}$. With this we can represent the connectivity pattern by a weight matrix $W$ having elements $W_{\mathrm{jir}}$. The connectivity pattern reveals the network architecture. The following two steps determine the activity of a unit within the output layer.

Step 1: Obtain the overall weighted input $X_{\mathrm{j}}$ by:

$$
X_{j}=\sum_{i} y i W_{j i}
$$

where $y_{i}$ is the level of activity in the preceding layer and $W_{\mathrm{ji}}$ as stated earlier is the weight of connection between two units $U_{\mathrm{j}}$ and $U_{\mathrm{i}}$.

Step 2: Compute the activity of $y_{\mathrm{i}}$ using sigmoid function of the overall weighted input. The sigmoid function commonly used is given by:

$$
y_{i}=\left(1+e^{-x_{j}}\right)^{-1}
$$

On completion of the activities of all output units the network computes the error $\mathrm{E}$ which is defined by:

$$
E=\frac{1}{2} \sum_{i}\left(y_{i}+0_{i}\right)^{2}
$$

where $y_{\mathrm{i}}$ is the top layer $j$ th unit activity level and $o_{\mathrm{i}}$ is the desired output of the unit $j$. 


\subsection{EMD-ANN Model}

We now present a hybrid (EMD-ANN) algorithm for forecasting electric load of the proposed model based on a three stage adaptive neural network paradigm. The first stage is the decomposition of the time series data into several intrinsic mode functions (IMFs) by applying the EMD extraction process described in Section 2.1. The second stage, we built separate IMF-ANN for each of the IMFs obtained in stage 1 as inputs for this model and the last stage, stage 3, we combine the prediction of these EMDIMF into one which is the prosed EMD-ANN model and make forecast with this. These three stages are further outlined below and shown in Figure 3.

Step1: Decompose the historical load data into several IMFs by using the EMD sifting process. Normalized all IMFs obtained, which are the inputs for the IMF-ANN model into a scalable range. For this study we use the following for the normalization:

$$
X_{\text {norm }}=\frac{\left(X_{i}-X_{\min }\right)}{\left(X_{\max }-X_{\min }\right)}
$$

where

$X_{i}$ are the actual values of the historical load data to be normalized

$X_{\text {norm }}$ are the normalized values of $\mathrm{X}$

$X_{\max }$ is the maximum value of $X$ in each IMF column

$X_{\min }$ is the minimum value of $\mathrm{X}$ in each IMF column

Step 2: Using the calculated IMFs and residue component as inputs develop separate models designated as IMF-ANN model for each

Step 3: Combine the predictions from each of these IMFANN models, to obtain the proposed model. Before forecasting with the model, obtain back the actual vales of the original series by:

$$
X_{i}=\left[X_{n o r m} *\left(X_{\max }-X_{\min }\right)\right]+X_{\min }
$$

This is obtained from equation (2.7).

\section{Implementation of the Model}

Nigeria has a well-established power utility concern called Power Holding Company of Nigeria (PHCN) and this company has offices in all the thirty-six states of the federation with its national headquarters situated in the capital

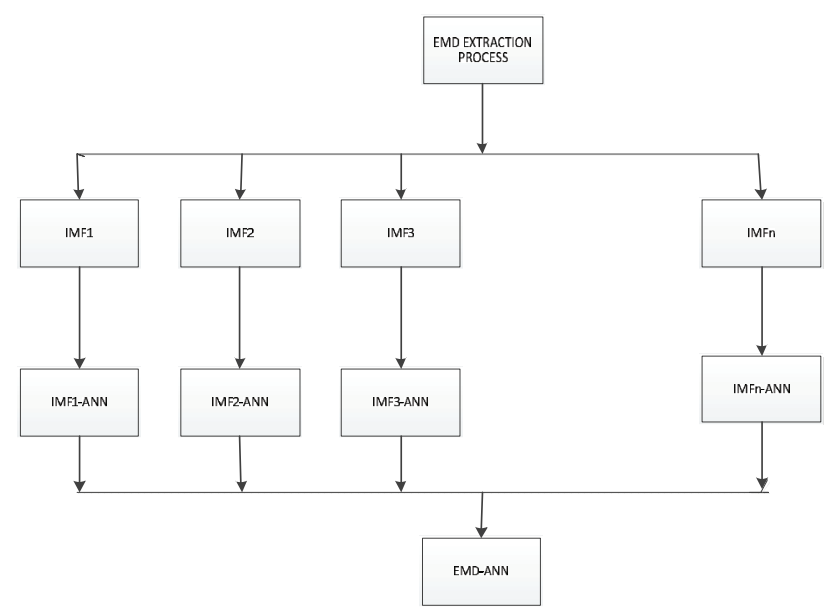

Figure 3. Pictorial Presentation of Proposed model.

city Abuja. The data for this study was collected from the business office of PHCN, Bida and it is the daily peak load demand for Bida area. The data covers the period January $1^{\text {st }}, 2012$ to December $31^{\text {st }}$, 2012. Figure 4 shows the daily peak load demand for Bida area. We divided the data sets into two, the first set comprises of $70 \%$ of the whole set and used them for training the model, while the last set is made up of $30 \%$ used for testing.

\subsection{Measure of Forecast Accuracy}

In order to effectively determine the forecast accuracy and thereafter ascertain whether the proposed model is better than the conventional neural network model for peak load forecasting, we used one index for evaluating the models and this is the mean absolute percentage error (MAPE), and the formula for computing this is:

$$
\text { MAPE }=\frac{1}{N} \sum_{i}^{N} \frac{\left(y_{\text {actual }}-y_{\text {forecast }}\right)}{y_{\text {actual }}} * 100 \%
$$

where

$N$ is the number of periods of time in the dataset

$y_{\text {actual }}$ are the actual values of $y$ in the dataset

$y_{\text {forecast }}$ are the forecasted values of $y$ in the dataset.

For evaluation purposes, a lower value of this index above is a measure that the forecast accuracy is better.

\subsection{Discussion of Results}

We tried out the proposed model on the historical load data obtained from PHCN, Bida, as well as using conventional neural network model on the same data. We obtained one day ahead forecast for both models for 


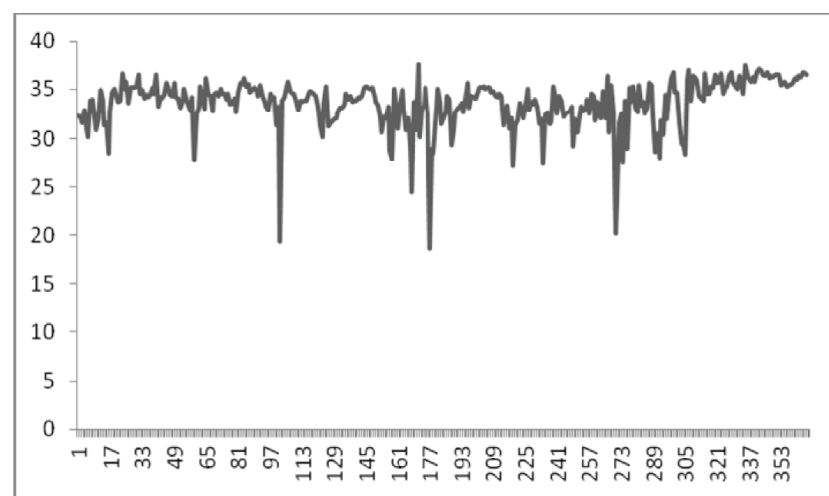

Figure 4. Electric Peak Load demand profile for Nigeria from January 1st to December 31st, 2012.

the whole year January $1^{\text {st }}, 2012$ to December 31 $1^{\text {st }}, 2012$. The results obtained are presented in Figure 5, 6, 7, Table 1 and 2.

We equally tried to forecast for weekdays ( Monday, Tuesday, Wednesday, Thursday and Friday) using the two models with $70 \%$ of the data set for training and $30 \%$ for testing. The results obtained are presented Figure 8, 9 and Table 3.

It is obvious from the results that the proposed EMDANN peak load forecasting model outperforms the conventional BPNN in terms of forecasting accuracy as it yields a lower MAPE whether for the whole year model or for the weekday model. Further we observe from Table 2 that of the fifteen Mondays in the forecast horizon for the whole year model the proposed EMD-ANN model gave better forecast result in eight of these while the conventional BPNN model only out classed in the proposed model in just five of these while two points were evenly forecasted. Also the result is an indication that the EMD extraction process is a good data pre-processing technique as it is its data smoothening ability that assisted the proposed model to achieve a better forecast accuracy. This is due to the fact that all the extracted IMFs and residue component are stationary.

\section{Conclusion}

A new approach to forecasting Electric Peak load has been presented in this paper. The model developed combines EMD and ANN. The results from this model are compared with that of a conventional BPNN model. The main idea behind this model is to decompose the historical data into several IMFs and a residue component using the EMD
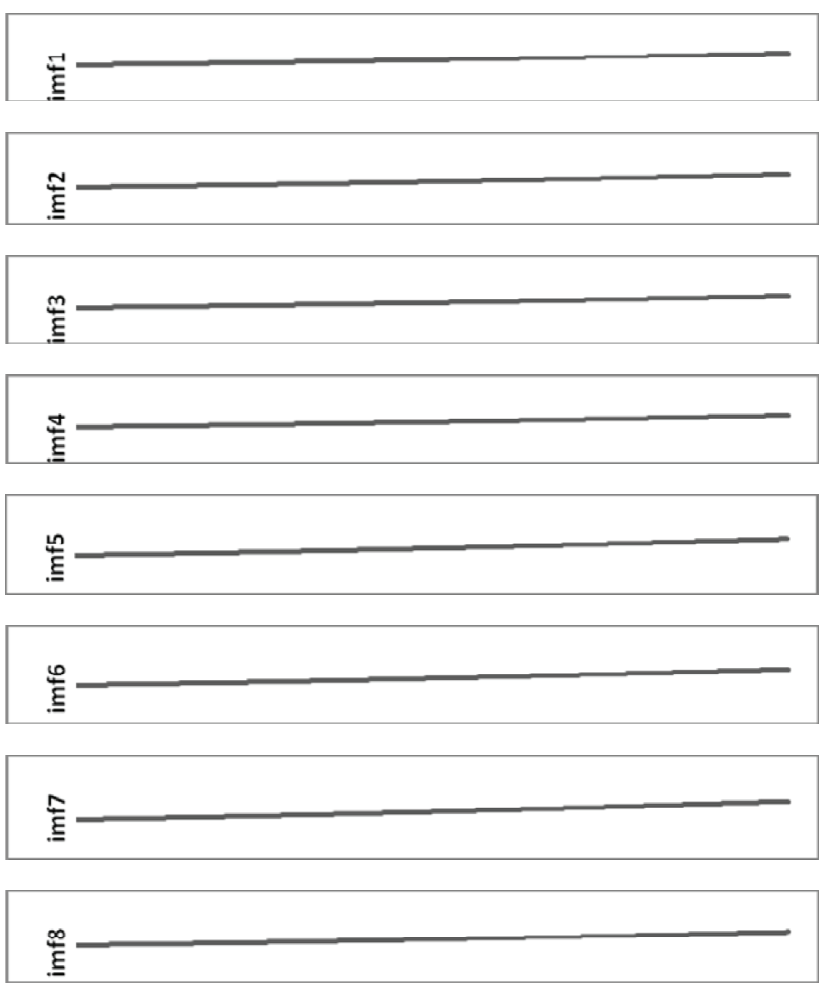

\section{要学}

Figure 5. The Extracted IMFs and Residue Component.

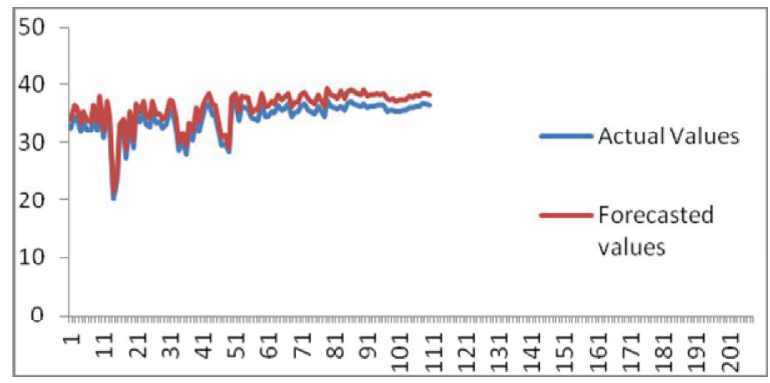

Figure 6. Actual and forecasted values for whole year.

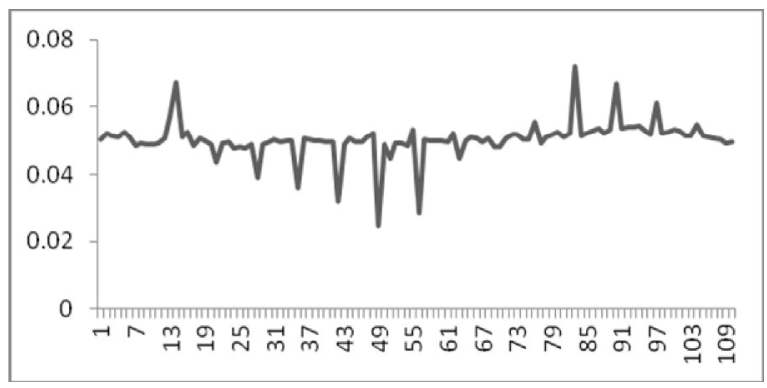

Figure 7. Error Curve for whole year. 
extraction process. This makes the time series more stationary likewise the forecast. Statistical analyses carried out on the historical data obtained from PHCN, Bida is an indication that the proposed EMD-ANN Electric Peak load forecasting model yields very accurate result over the conventional neural network model and as such can be adopted for peak load forecasting by utility concern where conventional neural network or other models are used.

\section{Acknowledgement}

We would like to express our appreciation to the following persons Engr. Mohammed Gaya Sani of the department of Electrical Engineering, Universiti Teknologi, Malaysia for his assistance in developing the MATLAB code for this research work, Engr. Eze O.N.E. and Aminu Maiangwar of PHCN, Bida for their assistance in obtaining the data used for this research work. We equally wish to thank the Ministry of Higher Education (MOHE), Malaysia for providing funds for this research through research grant with vote number $02 \mathrm{H} 52$ and the management of Universiti Teknologi Malaysia for allowing us use their facilities for this research.

Table 1. Forecast Accuracy for whole year model

\begin{tabular}{cccc}
\hline & EMD-ANN & Conventional BPNN & \% Improvement \\
\hline MAPE & 5.03 & 5.15 & 2.3 \\
\hline
\end{tabular}

\section{References}

1. Osofisan P B, and Nwaeke C N (2010). Application of Adaptive Neural Network (ANN) for short term load forecasting: case study on National Control Centre (PHCN), Oshogbo, Osun State, Nigeria, Journal of Engineering and Applied Sciences, vol 5(2), 78-83.

2. Igbinovia S O, and Odiase F O (2009). Electric energy pricing in a deregulated economy: a case study of delta power plant, delta state, Nigeria, Journal of Engineering and Applied Sciences, vol 4, No. 2, 145-151.

3. Fadare D A, and Dahunsi O A (2009). Modeling and forecasting of short-term half-hourly electric load at the university of Ibadan, Pacific Journal of Science and Technology, vol 10, No.2, 471-478.

4. Adepoju G A, Ogunjuyigbe S O A et al. (2007). An Application of Neural Network to load Forecasting in Nigerian Electrical Power System, The Pacific Journal of Science and Technology, vol 8, No. 1, 68-72.

5. Adenikinju A (2005). Analysis of the cost of Infrastructure failures in a developing economy: The case of the electricity sector in Nigeria, African Economic Research Consortium, Nairobi, Kenya, AERC Research Paper 148.

6. Huang N E, Shen Z et al. (1998). An empirical mode decomposition and the Hilbert spectrum for nonlinear and non-stationary time series analysis, Proceedings of the Royal Society of London A, vol 454, No. 1971, 903-995.

7. Okolobah V, and Ismail Z (2013a). Forecasting Peak load demand by ARIMA and EMD method, Archives Des Science, vol 66, No.1, 1-14.

Table 2. Forecast results for all Mondays in the testing set

\begin{tabular}{|c|c|c|c|c|c|}
\hline Date & Actual Value & EMD-ANN forecasted Value & \% Error & Conventional BPNN & $\%$ Error \\
\hline & 33.7 & 35.47 & 5.25 & 35.40 & 5.05 \\
\hline & 35.4 & 37.19 & 5.06 & 37.24 & 5.20 \\
\hline & 33.8 & 35.48 & 4.97 & 35.50 & 5.03 \\
\hline & 35.4 & 37.08 & 4.75 & 37.16 & 4.97 \\
\hline & 32.4 & 34.02 & 5.00 & 34.02 & 5.00 \\
\hline & 32 & 33.58 & 4.94 & 33.60 & 5,00 \\
\hline & 29.4 & 30.90 & 5.10 & 30.90 & 5.10 \\
\hline & 36.1 & 37.85 & 4.85 & 37.91 & 5.01 \\
\hline & 34.6 & 36.31 & 4.94 & 36.33 & 5.00 \\
\hline & 34.5 & 36.24 & 5.04 & 36.74 & 6.49 \\
\hline & 35.8 & 36.76 & 2.68 & 37.58 & 4.97 \\
\hline & 36.3 & 38.23 & 5.31 & 38.12 & 5.01 \\
\hline & 36.5 & 38.39 & 5.18 & 38.31 & 4.96 \\
\hline & 35.6 & 37.43 & 5.14 & 37.38 & 5.00 \\
\hline & 36.5 & 38.31 & 4.96 & 38.29 & 4.90 \\
\hline
\end{tabular}




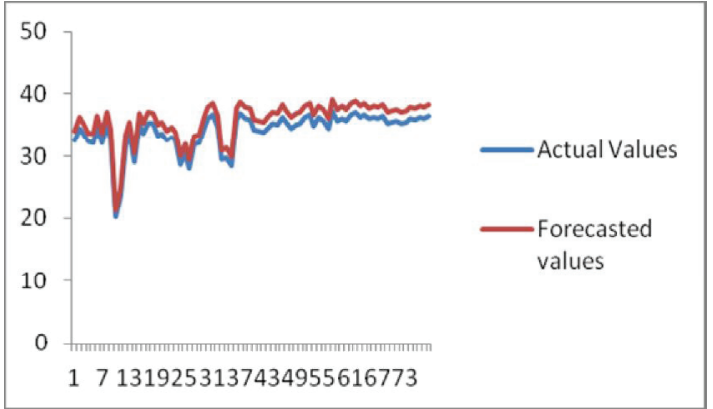

Figure 8. Actual and Forecasted values for weekday load.

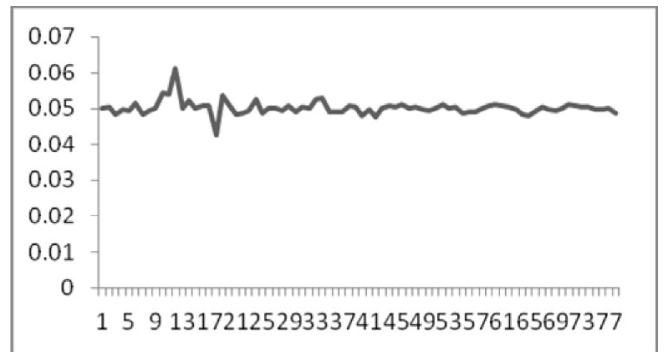

Figure 9. Error curve for weekday load.

Table 3. Forecast Accuracy for weekday model

\begin{tabular}{cccc}
\hline & EMD-ANN & Conventional BPNN & \% Improvement \\
\hline MAPE & 5.02 & 5.11 & 1.8 \\
\hline
\end{tabular}

8. Kutbatsky V, Sidorov D et al. (2011). Hybrid model for shortterm forecasting in electric power system, International Journal of Machine Learning and Computing, vol 1(2), 138-147.

9. Okolobah V, and Ismail Z (2013b). Electricity load analysis based on empirical mode decomposition, $4^{\text {th }}$ International
Graduate Conference on Engineering, Science and Humanities, UniversitiTeknologi Malaysia, 965-972.

10. Guo Z, Zhao W et al. (2012). Multi-step forecasting for wind speed using a modified EMD-based Artificial Neural Network model, Journal of Renewable Energy, vol 37(1), 241-249.

11. Zhu Z, Sun Y et al. (2007). Hybrid of EMD and SVMs for short-term load forecsting, 2007 IEEE International conference on control and automation, Guangzhou, China, 1044-1047.

12. Ran L, and Yue W (2008). Short-term wind speed forecasting for wind farm based on empirical mode decomposition, IEEE Explore, 2521-2525.

13. Flandrin P, and Goncalves P (2004). Empirical mode decomposition as a filter bank, IEEE Signal Processing letters, vol 11, No. 2, 112-114.

14. Chen Q, Huang $\mathrm{N}$ et al. (2006). A B-spline approach for empirical mode decomposition, Advances in Computational Mathematics, vol 24, 171-195.

15. Rilling G, Flandrin P et al. (2003). On empirical mode decomposition and its algorithms, IEEE-EURASIP workshop on Nonlinear Signal and image Processing, Grado (I).

16. Huang N E, Wu M L et al. (2003). A confidence limit for the empirical mode decomposition and Hilbert Spectrum analysis, Proceedings of the Royal Society of London, A459, 2317-2345.

17. Nunes J C, Bouaoune Y et al. (2003). Image analysis by bidimensional empirical mode decomposition, Image and Vision Computing, vol 21(12), 1019-1026.

18. Jha G K (2004). Artificial Neural Networks, Indian Agricultural Research Institute, 1-10.

19. Zhang X, Lai K K et al. (2008). A new approach for crude oil price analysis based on Empirical Mode Decomposition, Elsevier Journal of Energy Economics, vol 30, 905-908. 